\title{
Elderly at time of COronaVIrus disease 2019 (COVID-19): possible role of immunosenescence and malnutrition
}

\author{
Leonardo Bencivenga $(\mathbb{D}$ - Giuseppe Rengo $\mathbb{D}$ • Gilda \\ Varricchi (10)
}

Received: 29 April 2020 / Accepted: 15 June 2020 /Published online: 23 June 2020

(C) American Aging Association 2020

\begin{abstract}
The unprecedented COVID-19 pandemic is rapidly and unpredictably evolving and the majority of deaths are occurring in older people. A partial description of the magnitude of the scenario is provided by numbers and statistics, which probably underestimate the ongoing tragedy. In the present opinion paper, we have focused our attention on the evidence of the relationship among malnutrition, immunosenescence, and the higher morbidity and mortality in elderly patients. In particular, we propose the intriguing hypothesis that
\end{abstract}

L. Bencivenga $\cdot$ G. Rengo $(\bowtie) \cdot G$. Varricchi

Department of Translational Medical Sciences, University of

Naples Federico II, Via Sergio Pansini 5, 80131 Naples, Italy

e-mail: giuseppe.rengo@unina.it

L. Bencivenga

Department of Advanced Biomedical Sciences, University of Naples Federico II, Naples, Italy

G. Rengo

Istituti Clinici Scientifici Maugeri SpA Società Benefit (ICS Maugeri SpA SB), Telese Terme, BN, Italy

G. Varricchi

Center for Basic and Clinical Immunology Research (CISI), University of Naples Federico II, Naples, Italy

G. Varricchi

WAO Center of Excellence, Naples, Italy

G. Varricchi

National Research Council (CNR), Institute of Experimental Endocrinology and Oncology “G. Salvatore” (IEOS), Naples, Italy correction of nutritional deficits may attenuate the age-dependent alterations of the innate and adaptive immune system which participate in the increased susceptibility and worse outcome observed in the elderly COVID-19 patients.

Keywords Pandemic COVID-19.

Immunosenescence $\cdot$ Malnutrition $\cdot$ Elderly

After the quick global spread outside China, where in Wuhan, in December 2019, the novel severe acute respiratory syndrome coronavirus 2 (SARS-CoV-2) was first identified as the aetiologic agent of a new type of viral pneumonia, the COronaVIrus Disease 19 (COVID-19) was declared a pandemic by the World Health Organization (WHO) on March 11, 2020 (World Health Organization 2020).

The scenario is rapidly and unpredictably evolving, and almost all countries are paying a very high price in terms of morbidity and mortality, including frontline health workers. The war against the invisible enemy is upsetting the global population and undermining the economic balance of entire production systems.

As expected, it became immediately evident that the majority of deaths from COVID-19 occurs in the elderly, especially in those suffering two or more comorbidities, with cardiovascular diseases, diabetes mellitus, chronic obstructive pulmonary disease, malignancy and chronic kidney disease being the most frequent (Emami et al. 2020). Additional factors worsening the COVID-19 
emergency within geriatric population may include intrinsic multidimensional features characterizing older subjects, such as disabilities, cognitive and mood disorders, polypharmacotherapy, social isolation, nutritional deficits and extrinsic factors detectable in the widespread ageism, the generalized lack of geriatric culture and alleged errors in the management of long-term care facilities.

Importantly, advanced age and comorbidities are related to impaired nutritional status and a recent meta-analysis indicates the prevalence of high malnutrition risk in Europe to be 28\%, $17.5 \%$ and $8.5 \%$, for hospital, residential care and community settings, respectively (Leij-Halfwerk et al. 2019). Nutritional deficits arise from malabsorption, increased nutrient losses and augmented metabolic demands, which are frequent in the elderly, but also from reduced dietary intake. Malnutrition is a major geriatric syndrome due to multifactorial etiology, characterized by muscle wasting and weight loss, which is strongly related to frailty and negative outcomes (Volkert et al. 2019). It is important to underline that malnutrition constitutes an underrecognized and undertreated condition, therefore, the aforementioned percentage may be underestimated.

As widely ascertained, malnutrition negatively affects the outcomes of elderly individuals, irrespective of the clinical setting; malnourished patients experience multiple changes in physiological functions involving several systems such as cardiorespiratory, gastrointestinal and musculoskeletal. Nutritional status exerts a relevant impact on the immune system of older people through impairment in cellmediated immunity, cytokine production and phagocytic function. In addition, aging per se is characterized by a process of progressive dysfunction of several compartments of the immune system, named immunosenescence, which includes smoldering inflammation and immunodeficiency. The complex interplay between immunity and nutrition in aging, due to alterations of both innate and adaptive immune responses, is clinically relevant and negatively impacts antibody production, response to vaccination and susceptibility to infections (Alam et al. 2019; Varricchi et al. 2020). Notably, the altered response to vaccination should be seriously considered and evaluated by randomized control trials (RCTs) when an efficient vaccine against SARSCoV-2 will be available.
The management of critical COVID-19 patients is particularly complex given the lack of antiviral agents specific for SARS-CoV-2 and the incomplete understanding of the pathophysiologic mechanisms of this disease. After a variable incubation period, SARS-CoV-2 causes mild to severe symptoms: in this stage, the activated innate and adaptive immune system of the majority of patients overcomes the infection. This is associated with the production of high affinity IgG anti-virus antibodies resulting from the efficient cooperation of $\mathrm{CD}^{+} \mathrm{T}_{\mathrm{FH}}$ cells and $\mathrm{B}$ cells. In addition, $\mathrm{CD}^{+} \mathrm{T}$ cells contribute to clear virus infected cells. It is important to emphasize that both arms of the immune system are altered in elderly (Varricchi et al. 2020).

Due to the age-dependent alterations of the immune system that may be implemented by nutritional deficits, a relevant percentage of elderly patients progresses to insidious systemic inflammation, mainly affecting the lung, heart, renal function and coagulation system. This second stage of hyperinflammation is characterized by massive production of pleiotropic cytokines (e.g., IL-6) by lung resident (e.g., macrophages) and circulating immune cells (Aiello et al. 2019).

Based on the latter findings, a treatment with tocilizumab (TCZ), a monoclonal antibody anti-IL6 receptor (IL-6R), effective for the treatment of rheumatoid arthritis, is under evaluation in COVID-19 patients (Di Giambenedetto et al. 2020). IL-6 is a cytokine playing a crucial role in COVID-19-induced cytokine storms. However, even if promising, there are few real-life data about the efficacy of TCZ on the inflammatory activity in COVID-19 patients and several RCTs are underway (NCT04332913, NCT04377659, NCT04377750).

There is compelling evidence that several nutrients, including vitamins (A, B group, C, D, E) and minerals (zinc, iron, selenium, magnesium and copper), exert a key role in supporting the innate and adaptive immune systems. Regarding innate immunity, micronutrients contribute to the development and integrity of physical barriers, participate to neutrophil/macrophage maturation and functions, and play a role in modulating inflammation. Additionally, adaptive immunity is influenced by nutritional deficits, especially lymphocyte maturation and differentiation, memory cell generation, and cytokine and antibody production (Calder et al. 2020). 
Nutritional deficits are most prevalent in older populations, thus contributing to weakening of the immune system. The aging process is characterized by enhanced catabolism and reduced anabolism, thus elderly patients would benefit from increased protein intake, especially in severe systemic conditions, such as wasting syndromes also associated with COVID-19 (Deutz et al. 2014). Of note, nutritional support has been shown to improve immune responses in older malnourished patients (Pansarasa et al. 2019; Aiello et al. 2019).

Zinc deficit is common in malnourished elderly individuals and it is related to increased risk of infections. Accordingly, adequate zinc support counteracts the alteration in the immune response through the enhancement of both total number and function of $\mathrm{T}$ cells (Barnett et al. 2016). Moreover, zinc supplementation has been reported to increase natural killer cell (NK) activity via enhancement of perforin expression. Deficiency of this trace element is indeed related to reduced NK cytotoxicity (Rolles et al. 2018). Vitamin A is a key factor for development of the immune system and modulates immune processes. It is essential for epithelial and mucosal barrier generation and it plays a role in immune cell homeostasis, differentiation and function. Further, vitamin A also contributes to support the efficacy of $\mathrm{T}$ cell-based viral vaccines (Huang et al. 2018).

Vitamin E supplementation has been reported to stimulate $\mathrm{T}$ cell function in both pre-clinical and clinical studies, and confers protection against upper respiratory tract infection in the elderly (Lee and Han 2018). Recent evidence supports a role of probiotics in modulating the immune system, with probiotic supplementation recognized as an effective nutritional intervention (Pae et al. 2012).

Vitamin D modulates several aspects of the innate and adaptive immune system and its deficiency is highly prevalent in the elderly worldwide. Vitamin D supplementation appears to reduce the risk of infections through cathelicidins and defensins that can reduce both viral replication and the production of pro-inflammatory cytokines. Moreover, vitamin D promotes differentiation of monocytes to macrophages and plays a role in the process of antigen presentation. On the basis of these promises, it has been proposed to administer high doses of vitamin D in COVID-19 patients (Jakovac 2020; Grant et al. 2020). A recent trial has assessed the effects of vitamin D supplementation on influenza vaccine response in elderly vitamin D-deficient subjects (Goncalves-Mendes et al. 2019). Surprisingly, no effect has been detected on antibody production, thus concurring to underline the complex metabolic-immunological substrate of the geriatric population. The latter aspects need to be considered whenever a vaccine against the SARS-CoV-2 will be available.

Vitamin $\mathrm{C}$ influences immunity through regulation of innate $\mathrm{B}$ and $\mathrm{T}$ cell proliferation and differentiation, their migration to sites of infection, and promoting antimicrobial activity and antibody production. Since vitamin $\mathrm{C}$ tends to be depleted during infections, particularly in patients developing respiratory distress syndrome (ARDS), clinical trials to investigate the efficacy of intravenous vitamin $\mathrm{C}$ in COVID-19 patients with ARDS have started (Carr 2020). Beside the relevant role of vitamin $\mathrm{C}$ in curbing oxidative stress-induced tissue damage, this novel line of investigation is supported by a previous observation which indicates a reduced pneumonia severity in older patients supplemented with vitamin $\mathrm{C}$, especially in those with lower baseline concentrations (Hemilä and Louhiala 2013).

Lymphopenia, a negative prognostic factor in COVID-19 critical patients, is also a marker of malnutrition, and it has recently been suggested that low prealbumin level could be related to ARDS in SARSCoV-2 infected patients (Wu et al. 2020).

Based on the aforementioned evidence, we would like to underline that immunosenescence and malnutrition can synergistically contribute to the augmented susceptibility and worse outcome of aged people to SARS-CoV-2. The assessment of nutritional and immune status, particularly in the elderly, could be important to identify patients at high risk. Clinical trials evaluating the efficacy of nutritional support in these patients are needed to verify the impact on mortality rates in the elderly.

Funding information Dr. Leonardo Bencivenga has been supported by a research grant provided by the Cardiopath $\mathrm{PhD}$ program.

\section{Compliance with ethical standards}

Conflict of interest The authors declare that they have no conflict of interest. 


\section{References}

Aiello A, Farzaneh F, Candore G, Caruso C, Davinelli S, Gambino $\mathrm{CM}$, et al. Immunosenescence and its hallmarks: how to oppose aging strategically? A review of potential options for therapeutic intervention. Front Immunol. 2019;10:2247. https://doi.org/10.3389/fimmu.2019.02247.

Alam I, Almajwal AM, Alam W, Alam I, Ullah N, Abulmeaaty M, et al. The immune-nutrition interplay in aging - facts and controversies. Nutr Heal Aging. 2019;5:73-95.

Barnett JB, Dao MC, Hamer DH, Kandel R, Brandeis G, Wu D, et al. Effect of zinc supplementation on serum zinc concentration and $\mathrm{T}$ cell proliferation in nursing home elderly: a randomized, double-blind, placebo-controlled triall. Am J Clin Nutr. 2016;103:942-51. https://doi.org/10.3945 /ajen.115.115188.

Calder PC, Carr AC, Gombart AF, Eggersdorfer M. Optimal nutritional status for a well-functioning immune system is an important factor to protect against viral infections. Nutrients. 2020;12:1181. https://doi.org/10.3390/nu12041181.

Carr AC. A new clinical trial to test high-dose vitamin $\mathrm{C}$ in patients with COVID-19. Crit Care. 2020;24:133. https://doi. org/10.1186/s13054-020-02851-4.

Deutz NEP, Bauer JM, Barazzoni R, Biolo G, Boirie Y, BosyWestphal A, et al. Protein intake and exercise for optimal muscle function with aging: recommendations from the ESPEN expert group. Clin Nutr. 2014;33:929-36. https://doi.org/10.1016/j.clnu.2014.04.007.

Di Giambenedetto S, Ciccullo A, Borghetti A, et al. Off-label use of tocilizumab in patients with SARS-CoV-2 infection. J Med Virol. 2020. https://doi.org/10.1002/jmv.25897.

Emami A, Javanmardi F, Pirbonyeh N, Akbari A. Prevalence of underlying diseases in hospitalized patients with COVID-19: a systematic review and meta-analysis. Arch Acad Emerg Med. 2020;8:e35.

Goncalves-Mendes N, Talvas J, Dualé C, Guttmann A, Corbin V, Marceau G, et al. Impact of Vitamin D supplementation on influenza vaccine response and immune functions in deficient elderly persons: A randomized placebo-controlled trial. Front Immunol. 2019;10. https://doi.org/10.3389 /fimmu.2019.00065.

Grant WB, Lahore H, McDonnell SL, et al. Evidence that vitamin D supplementation could reduce risk of influenza and COVID-19 infections and deaths. Nutrients. 2020;12:988. https://doi.org/10.3390/nu12040988.

Hemilä H, Louhiala P. Vitamin C for preventing and treating pneumonia. Cochrane Database Syst Rev. 2013, 2013.
Huang Z, Liu Y, Qi G, Brand D, Zheng S. Role of vitamin A in the immune system. J Clin Med. 2018;7:258. https://doi. org/10.3390/jcm 7090258 .

Jakovac H. COVID-19 and vitamin D-Is there a link and an opportunity for intervention? Am J Physiol Endocrinol Metab. 2020;318:E589. https://doi.org/10.1152 /ajpendo.00138.2020.

Lee GY, Han SN. The role of vitamin E in immunity. Nutrients. 2018;10.

Leij-Halfwerk S, Verwijs MH, van Houdt S, Borkent JW, Guaitoli PR, Pelgrim T, et al. Prevalence of protein-energy malnutrition risk in European older adults in community, residential and hospital settings, according to 22 malnutrition screening tools validated for use in adults $\geq 65$ years: a systematic review and meta-analysis. Maturitas. 2019;126:80-9.

Pae M, Meydani SN, Wu D. The role of nutrition in enhancing immunity in aging. Aging Dis. 2012;3:91-129.

Pansarasa O, Pistono C, Davin A, Bordoni M, Mimmi MC, Guaita A, et al. Altered immune system in frailty: genetics and diet may influence inflammation. Ageing Res Rev. 2019;54:100935.

Rolles B, Maywald M, Rink L. Influence of zinc deficiency and supplementation on NK cell cytotoxicity. J Funct Foods. 2018;48:322-8. https://doi.org/10.1016/j.jff.2018.07.027.

Varricchi G, Bencivenga L, Poto R, Pecoraro A, Shamji MH, Rengo $\mathrm{G}$. The emerging role of $\mathrm{T}$ follicular helper (TFH) cells in aging: influence on the immune frailty. Ageing Res Rev. 2020;101071:101071. https://doi.org/10.1016/j. arr.2020.101071.

Volkert D, Beck AM, Cederholm T, Cereda E, Cruz-Jentoft A, Goisser S, et al. Management of Malnutrition in older patients - current approaches, evidence and open questions. J Clin Med. 2019;8:974. https://doi.org/10.3390 /jcm8070974.

World Health Organization W (2020) WHO Director-General's opening remarks at the media briefing on COVID-19 - 11 March 2020. In: WHO Dir. Gen. speeches. https://www.who. $\mathrm{int} / \mathrm{dg} /$ speeches/detail/who-director-general-s-openingremarks-at-the-media-briefing-on-covid-19\%2D\%2D-11march-2020. Accessed 11 Apr 2020.

Wu C, Chen X, Cai Y, Xia J', Zhou X, Xu S, et al. Risk factors associated with acute respiratory distress syndrome and death in patients with coronavirus disease 2019 pneumonia in Wuhan. China JAMA Intern Med. 2020. https://doi. org/10.1001/jamainternmed.2020.0994.

Publisher's note Springer Nature remains neutral with regard to jurisdictional claims in published maps and institutional affiliations. 